Methodology In our randomized pilot study, ten patients were tested using FRD staining solution. In all 10 patients HPV Test, Pap Smear, and Colposcopy were also performed. These four methods were evaluated in regard to the histopathological findings of the targeted biopsy.

Results The sensitivity and specificity of FRD staining solution were $100 \%$ and $50 \%$, respectively.

Conclusion The advantage of the FRD method is that the results are immediate. Another benefit of this test is that it can predict the location of cervical dysplasia both on the cervix and in the canal itself.

Further study could be useful to check if overall accuracy for screening is improved when FRD is used as a co-test with HPV testing.

Disclosures None.

\section{Endometrial cancer}

\section{SURGICAL MANAGEMENT OF GYNECOLOGIC CANCERS DURING THE COVID-19 PANDEMIC}

${ }^{1}$ Nazila Shokri, ${ }^{1}$ Yekta Parsa, ${ }^{2}$ Hamidreza Niazkar, 'Zahra Naeiji, ${ }^{1}$ Atefeh Moridi. ${ }^{1}$ Shahid Beheshti University of Medical Sciences; Obstetrics And Gynecology; ${ }^{2}$ Student Research Committee, Gonabad University of Medical Sciences, Gonabad, Iran

\subsection{6/ijgc-2020-ESGO.211}

Introduction/Background The COVID-19 pandemic brings about various challenges for surgeons in different fields. They should assess the risk-benefit of each surgery prior to the operation, and decide whether the surgery is beneficial for the patient or the surgery is delayable due to the risk of COVID19 infection. In this regard, gyneco-oncology surgeries are no exception. If the treatment is deferred, it may lead to the progression of the disease, affect the quality of life and patient's survival.

Case Reports In this article, we report and discuss three cases of gynecologic cancer including two cases of endometrial cancer and one case of cervical cancer in situ that referred to Mahdiyeh hospital, Tehran, Iran, during the COVID-19 pandemic.

Conclusion According to the centers for disease control and prevention (CDC) guidelines, the COVID-19 Polymerase Chain Reaction (PCR) must be performed for each patient before surgery. If the patient was positive for COVID-19, the surgery should be postponed for at least two weeks. If the test is negative and the patient is candidate for surgery, delay in surgery should be minimized and efforts should be made to discharge the patient earlier to reduce the contact of patient with health worker and other patients. All of these processes are to protect the cancer patient from COVID-19 infection. For the current situation of COVID-19 pandemic, risk assessment should be done carefully to identify whether the role of surgery is curative or palliative and how it may impact the life expectancy of the patient. Every cancer patient should be screened for possible infection before the surgery. During the surgery, measures should be taken to reduce the time of surgery and complications that my lead to ICU (intensive care unit) admissions. Discharging patients earlier after the surgery could also reduce the risk of infection.

Disclosures There is not any conflict of interest to be declared regarding the manuscript.

\section{A PILOT STUDY FOR THE VALIDATION OF SENTINEL LYMPH NODE BIOPSY WITH INDOCYANINE GREEN FLUORESCENCE METHOD IN EARLY ENDOMETRIAL CANCER AT FUNDACIÓN JIMÉNEZ DÍAZ UNIVERSITY HOSPITAL}

José García Villayzan.

\subsection{6/ijgc-2020-ESG0.212}

Introduction/Background Sentinel Lymph Node Biopsy is a technique developed to predict lymphatic involvement in patients with early endometrial cancer, decreasing the morbimortality associated with routine systematic lymphadenectomy and improving quality of life.

Methodology Main Objective: To determine the detection rate and negative predictive value of the Sentinel Lymph Node Biopsy by Immunofluorescence in patients with early endometrial cancer.

Secondary Objective: To determine the morbidity and mortality associated with Sentinel Lymph Node Biopsy in comparison to systematic lymphadenectomy

To determine the quality of life of the patients who only underwent Sentinel Lymph Node Biopsy in comparison to systematic lymphadenectomy

Method A descriptive observational study in patients with early endometrial cancer (FIGO stage I-II) for all histological types and grades, who underwent the Sentinel Lymph Node by immunofluorescence Technique and/or systematic

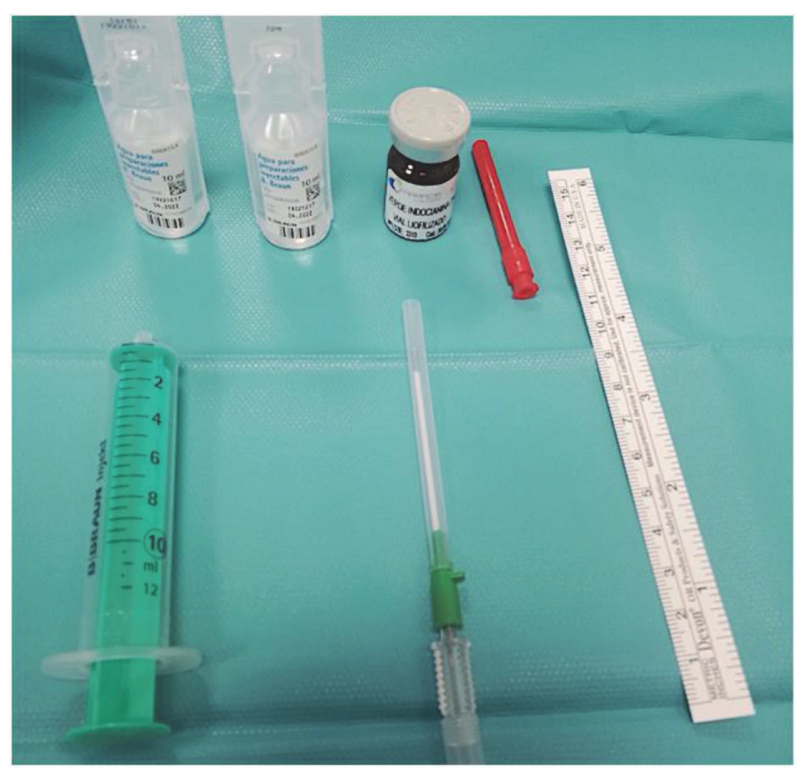

Abstract 585 Figure 1

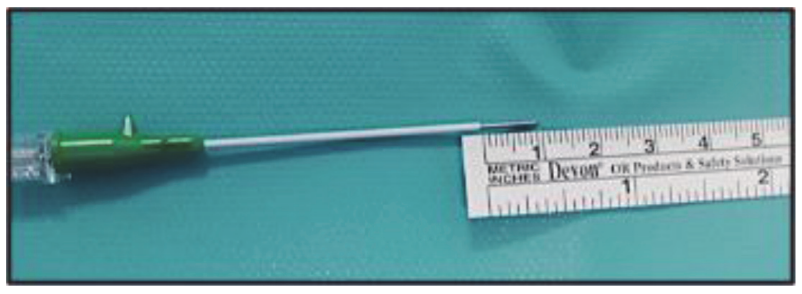

Abstract 585 Figure 2 
Abstract 585 Table 1 Comparison of the cases according to the learning curve and improvement of the technique

\begin{tabular}{llll}
\hline & First 10 patients & Last 8 patients & $\mathbf{p}$ \\
\hline AGE & $64,50 \pm 11,655$ & $64,25 \pm 9,94$ & $>0,05$ \\
BMI & $27,268 \pm 3,32$ & $27,20 \pm 4,57$ & $>0,05$ \\
N $^{\circ}$ SN MAPEADOS & $1(0-3)$ & $2(1-3)$ & 0,006 \\
N $^{\text {SN FALLADOS }}$ & $1(0-2)$ & $0(0-1)$ & 0,003 \\
N $^{\circ}$ TOTAL SN & $17(1-42)$ & $21(1-44)$ & $>0,05$ \\
REMOVE & & & \\
\hline
\end{tabular}

BMI: Body mass index $\left(\mathrm{kg} / \mathrm{m}^{2}\right)$, SN: Sentinel Node.

lymphadenectomy between June 2019 and March 2020 at the Fundación Jiménez Díaz University Hospital.

We used indocyanine green powder for injection, with a concentration of 25 milligrams (mg). We proceeded to dissolve it in 10 cubic centimeters (cc) of distilled water to avoid precipitation of the marker; obtaining a final concentration of $2.5 \mathrm{mg}$. After which, we injected $2 \mathrm{cc}$ of the prepared solution into the cervix at the 3 and 9 o'clock positions at a depth of $1 \mathrm{~cm}$ through Abbocath $\mathrm{N}^{\circ} 12 \mathrm{G}$ (figure 1).

Results Eighteen patients were included, analyzing a total of 26 sentinel nodes: 24 pelvic and 2 paraortic; and a total of 273 lymph nodes (sentinel and non-sentinel nodes): 83 right pelvic, 86 left pelvic and 104 paraortic. All nodes were negative for metastasis.

Global and bilateral detection rates were $77.78 \%$ and $50 \%$ respectively. The Negative Predictive Value and sensitivity were $100 \%$. No significant difference in morbimortality was found between performing only Sentinel Lymph Node technique or systematic lymphadenectomy; but the association with quality of life was significant, with better results for those who only underwent the sentinel lymph node technique versus systematic lymphadenectomy ( $0 \%$ vs $77 \%$ ).

However, we observed at the beginning of the study bilateral detection was very limited. This could have been due to a failure in the tracer injection technique in our first 10 patients. Since the standardization of the technique we obtained a considerable improvement in bilateral detection; $87.5 \%$ (before technical standardization 20\%). This supports the theory that technique is the most important factor in detection (Rossi, 2019). On the other hand, it is important to assess the learning curve, considered an independent factor that can influence the quality of the technique (table 1).

Conclusion The global and bilateral detection rates of the Sentinel Lymph Node Technique by immunofluorescence were $77.78 \%$ and $50 \%$ respectively, obtaining a Negative Predictive Value and Sensitivity of $100 \%$. Sentinel Node Biopsy is a valid technique to predict lymphatic affectation in early endometrial cancer, with lower morbimortality than systematic lymphadenectomy (figure 2).

Disclosures No conflict of interest to disclose.

\section{EARLY STAGE, LOW GRADE ENDOMETRIAL ADENOCARCINOMA IN REPRODUCTIVE AGED WOMEN. AN EVALUATION OF PATIENT PERSPECTIVES OF DIAGNOSIS, TREATMENT DECISION MAKING, MANAGEMENT, FERTILITY AND SURVIVORSHIP: A QUALITATIVE STUDY}

${ }^{1}$ Alina Roman, ${ }^{2}$ Michelle Peate, ${ }^{3}$ Yasmin Jayasinghe, ${ }^{4}$ Orla M Mcnally. ${ }^{1}$ The Royal Women's Hospital; Gynaecology Oncology, Melbourne Australia; ${ }^{2}$ The Royal Women's Hospital; The University of Melbourne, Melbourne Australia; ${ }^{3}$ The Royal Women's Hospital; The Royal Children's Hospital, Melbourne Australia; ${ }^{4}$ The Royal Women's Hospital; Peter Maccallum Cancer Centre, Melbourne Australia

\subsection{6/ijgc-2020-ESG0.213}

Background Endometrial cancer is rare in reproductive aged women. Standard surgical treatment can impact fertility. Nonstandard conservative management with progestins can be used in very specific populations to give women an opportunity for childbearing. ${ }^{1}$ The treatment experiences of this cohort have not been well studied, and unmet needs not identified or explored. This study aims to explore the experiences of reproductive aged women with early endometrial cancer across

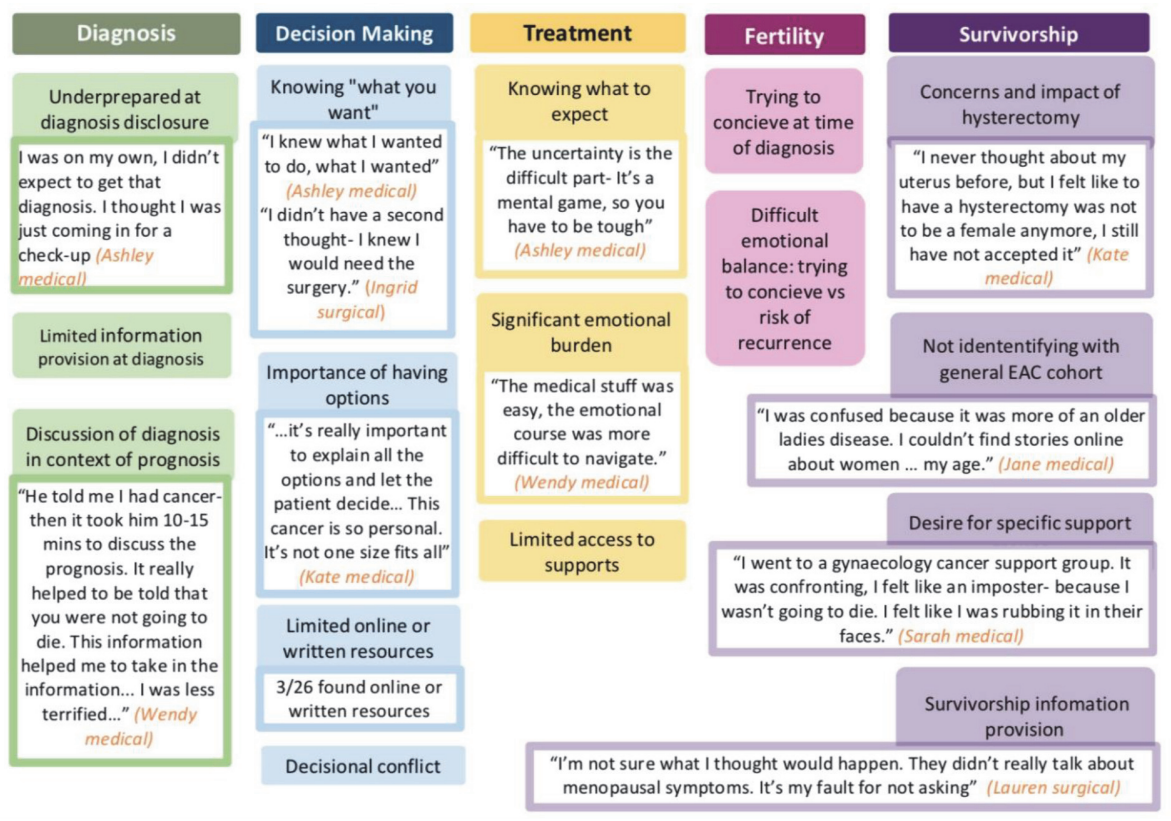

Abstract 589 Figure 1 Common themes identified in the experience of reproductive aged women with early EAC 